\title{
Semantic Web Standards and Ontologies for Legislative Drafting Support
}

\author{
Tommaso Agnoloni and Daniela Tiscornia \\ ITTIG-CNR, Via de' Barucci 20, Florence, Italy \\ \{agnoloni, tiscornia\}@ittig.cnr.it
}

\begin{abstract}
Machine readable open public data and the issue of multilingual web are open challenges promising to transform the relationship between citizens and European institutions. In this context the DALO\$1 project aims at ensuring coherence and alignment in the legislative language, providing law-makers with knowledge management tools to improve the control over the multilingual complexity of European legislation and over the linguistic and conceptual issues involved in its transposition into national laws. This paper describes the design and implementation activities performed on the basis of a set of parallel texts in different languages on a specific legal topic. Natural language processing techniques have been applied to automatically build lexicons for each language. Lexical and conceptual multilingual alignment has been accomplished exploiting terms position in parallel documents. An ontology describing entities involved in the chosen domain has been developed in order to provide a semantic description of terms in lexicons. A modular integration of such resources, represented in RDF/OWL standard format, allowed their effective and flexible access from a legislative drafting application prototype, able to enrich legal documents with terms mark-up and semantic annotations.
\end{abstract}

Keywords: Machine-Readable Open Data, Multilingual Legal Ontologies, Natural Language Processing, XML Authoring.

\section{Introduction}

A process of standardization of document formats, their unique identification and their annotation in machine readable format is being carried on in recent years under the impulse of web technologies. The envisaged goal is to make electronic documents interoperable among different systems, meaningfully interlinked in a web of documents and at the end more effectively accessible by human and artificial agents. Legal documents (norms, procedures, court decisions) due to their particular relevance in an effective and transparent democracy and their pervasiveness in all the fields of citizens' activities are natural candidate to be affected by such innovations. Moreover they are the outcomes of a complex, distributed and expensive bureaucratic machine whose reengineering in the light of

\footnotetext{
${ }^{1}$ DrAfting Legislation with Ontology-based Support.
} 
the availability of information technologies would produce huge potential benefits. Quality in European and national legislation is actually one of the main purposes of the current initiatives of the European Commission. In the Mandelkern report on better regulation [1] the need for a coordinated action by Member States was solicited to simplify the EU regulatory environment, to enhance the quality of EU legislation as well as to rationalise the transposition of European directives into national law. Coherence, interoperability and harmonization in the legislative knowledge of, and control over, the legal lexicon is therefore considered as a precondition for improving the quality of legislative language and for facilitating access to legislation by legal experts and citizens. In a multilingual environment and, in particular, in EU regulations, only the awareness of the subtleties of legal lexicon in the different languages can enable drafters to maintain coherence among different linguistic versions of the same text, as well as over their transposition in national laws. The DALOS project launched within the "eParticipation" framework of the EU Commission, tackled these problems from different point of views. Providing a legislative drafting environment able to produce legislative documents in a standard compliant interoperable XML format from the very beginning of their proposal. Integrating domain knowledge, conceptual and lexical resources in the editing tool in order to help and constrain the legislator to the use of shared and understandable (clear and effective) legal concepts within the legislation. Tackling the multilingual issue of European law providing cross-language and cross-culture shared and aligned legal concepts in order to favour uniform adoption and implementations of European regulations and directives in member states. Providing an initial nucleus of Linked Data on a specific domain capable of automatically contextualize, explain and rephrase relevant concepts through the semantic connection with relevant related resources on the web. The DALOS outcome have been essentially twofold: propose an authoring tool enabling the production of documents in standard reusable format annotated with respect to tags stored in an knowledge resource accessible from a user friendly environment. Propose and implement a methodology to encode, by extraction from a corpus of documents, the domain knowledge on a specific subject as the outcome of previous legislation and decisions on the subject to be reused in new document production and put in comparison with different linguistic versions for legal translation assistance and standardized for use in annotation of documents.

\section{Semantic Web Technologies and Legal Information}

The web as it has developed in the last decades is basically a web of interlinked documents. It is based over a few basic technologies URI, HTTP, HTML i.e. a standard for resources identification, a transfer protocol, a markup language enabling the display of documents in web browsers with typographical informations and hyperlinking to other documents. The huge potential of such innovation is evident in its everyday use. In recent years however the web is evolving from a global information space of interlinked documents to a web of data, i.e. a global 
distributed, flexible and queryable database. The linked data initiative [2] in fact aims at providing on the Web machine-readable data, complementing the human-readable documents that constitute the major part of the current Web. The goal is to allow computer-based agents to be much more efficient in assisting users and performing automated tasks on their behalf, thus realising the vision of the Semantic Web. In order to accomplish that an additional set of specification on the top of existing ones have been proposed and standardized by the W3C consortium in order to achieve standardized machine readable document formats (XML), a flexible relational model of data (RDF) and an ontology specification language $(\mathrm{OWL})$ enabling the definition of concepts and conceptual relations and the computation of conceptual inferences. XML (eXtensible Markup Language) provide the technology to ensure interoperability at the syntactic level, i.e. provides the mean to make documents readable and interchangeable among different systems using standardized and open document format for which numerous tools and applications have been developed and are widely available. Moreover any specific portion of documents or additional data on documents (metadata) can be structured in meaningful elements that can be read and interpreted by software applications. Syntactic interoperability is then ensured by the definition of common data structures, e.g. XML Schema to which a class of documents must comply (document validation). This already opens a scenario where a shift from documents to specific piece of information and from natural language to processable data takes place. Moreover technological barriers once format standards have been established and adopted on a large scale would be removed enabling a high potential of information exchange and reuse among different heterogeneous systems. RDF (Resource Descriprtion Framework) is the data model enabling the definition of typed links among data from different sources on the web just like in the current Hypertext web, hyperlinks establish (untyped) links among HTML documents. The RDF model encodes data in the form of subject, predicate, object triples. The subject and object of a triple are both URIs that each identify a resource, or a URI and a string respectively. The predicate specifies how the subject and the object are related. Thus RDF triples can be thought as link among items in different data sets analogous to hypertext links connecting one HTML document to another. RDF enables us to include in the documents machine understandable statements on relevant objects and their properties. An RDF triple can state for example that two resources, a person $\mathrm{A}$ and a document $\mathrm{B}$, both identified by a URI are related by the fact that $\mathrm{A}$ is the author of B. Such resources can be stored in different data sets exposed on the web thus creating a web of data. Semantic interoperability on the other hand aims at ensuring that the exchanged information is interpeted by communicating parties (human or artificial agents) with a shared meaning. This can be accomplished by the definition of semantic resources e.g. shared terminology, thesauri or ontologies ensuring that data elements are interpreted in the same way by explicitly tagging their content with reference to an encoded shared conceptualization. Semantic interoperability assets, e.g. taxonomies or code lists, are moreover particularly relevant in the multilingual and multicultural context 
of the European Union where data exchange faces the obstacles of linguistic and jurisdictional barriers 2. Actually semantic web technologies are also the key to tackle the challenge of a multilingual web by enabling the connection of language independent conceptualizations with lexical databases and semantic networks enabling language dependent access to resources. The RDF data model can in fact be used to publish data in a language-independent fashion and connect them with language dependent resources in order to enable users to access data in their own language. In the Legal Information field many initiatives have been promoted to implement semantic technologies in document production, storage and presentation. A standardization process in document formats both for the annotation of their textual content (specific normative text elements like heading, section, article, paragraph, reference or formatted text like tables, lists) and additional metadata (like subject classification, publication date, enacting authority, relatioship among acts) is being carried at National and European level. See 3 for an overview. At the European level the CEN/Metalex 3 initiative aims at becoming the standard interchange format for legal documents by implementing a metalevel of mapping among national document formats. The implementation of Semantic Web technologies in the Legislative field, focusing on the addition of computer processable information to legal documents, according to shared standards, facilitates for example the integration between:

- the production of documents and the management of their workflow

- the use of a shared consistent terminology among different implementation of the same legal concept in different legislative interventions on a topic or in different national implementations of a legislative provision.

- the distribution, access and reuse of the resulting documents and information for citizens (enhanced with such interlinked meta-information)

During the drafting phase for example machine processable data can be added to the various versions of a document in order to facilitate the subsequent retrieval of a specific version of a document and to keep track of its workflow. These same data (for instance the specification of the structure of a document, its authors, its life-cycle, etc.) can enrich the document when they are published, and can be used to provide information concerning the procedure through which the document has been produced. The approach of the Semantic Web facilitates on the one hand (from the "back office" side) legal drafting, the maintenance of legal sources for example enabling "point-in-time" access to in-force legislation or the implementation of normative references for easy navigation among cited documents, and the management of legislative workflows and procedures. On the other hand (on the "front office" side), the publicity and transparency of procedures and information, the dialogue between sub-national, national, and international institutions, and community stakeholders at every stage of the Legislation formation and debate. Machine readable data in fact, enabling the separation between content and presentation allow for straightforward implementation of

\footnotetext{
2 WWW.semic.eu

3 WWw.metalex.eu
} 
e-consultation applications during the whole legislative process. See also [4] for an overview of information technology impact on e-participation. According to the described scenario the DALOS project contribution have been essentially to:

1. Encode by extraction from a corpus of documents the domain knowledge on a specific subject as the outcome of previous legislation and decisions on the subject to be reused in new document editing and standardized for use in semantic annotation of content.

2. Tackle the multilinguality issue by providing a formal structure integrating language independent conceptualization encoded in a domain ontology connected with WordNet-like structured lexical resources providing language dependent lexicalizations.

3. Link multilingual lexicalizations of relevant concepts on a domain with their original context of use or definition in source document-fragments.

4. Integrate in a standard compliant XML authoring tool for legislative documents the access to external knowledge, lexical and documental multilingual resources for drafting support and document annotation from a non-technical user friendly environment.

In particular DALOS aims on one hand at ensuring that legal drafters and decision-makers have control over the legal language at national and European level, by providing law-makers with linguistic and knowledge management tools to be accessed in the legislative processes. On the other hand at guaranteeing open access to resulting data by implementing open document standards for favouring their subsequent reuse. The methodological approach proposed in DALOS for the design of the domain knowledge resource consists of three main activities:

1. semi-automatic term extraction from a set of selected documents by using NLP (Natural Language Processing) tools;

2. construction of a domain ontology;

3. integration of ontologies, lexical resources and documents.

The next three sections will focus on these points. Finally the integration of access instruments to the semantic resources from a Legislative XML drafting environment is described in Sect. 6]

\section{$3 \quad$ Building Aligned Lexicons}

The starting point for the construction of the lexicons is a selected corpus of documents on the domain of "protection of consumers" economic and legal interests, including Directives, Regulation and Case Law on the subject. From a methodological point of view, of great importance is the fact that the source documents for the construction of the resource are made available in parallel translations in the EU languages because of the obligation for European Institutions to publish directives in the national languages of the member states, 
making possible a subsequent automatic terms and concepts alignment. Domain lexicons in the four European languages (Italian, English, Spanish, Dutch) supported in DALOS have been constructed in a semiautomatic way applying Natural Language Processing techniques to the set of selected documents. For the extraction of Italian terms we used T2K (Text-to-Knowledge), a hybrid ontology learning system combining linguistic technologies and statistical techniques [5]. For the other languages two term extraction applications have been used. TermExtractor [6] offers a comprehensive package of algorithms for the selection of relevant terms from any text corpus. GATE 7, developed by the University of Sheffield, is a framework for Language Engineering (LE) applications which supports efficient and robust text processing. A key requisite for both the automatic tools was the possibility to keep track of the links to document fragments in the domain corpora from which each relevant term has been extracted. To this end a preliminary fragmentation of each document in its formal partitions (paragraphs of normative documents e.g. Part, Article etc.) have been performed. More important, this made possible the exploitation of the peculiarity of the domain corpus to be parallel. Interlingual alignment could in fact be automatically established exploiting the origin of extracted terms in different languages from parallel contexts. A particular treatment is reserved to terms identified by automatic tools to be definitions, i.e. terms preceding a definition in the directives. For this terms a translation in the different languages keeping the original meaning is guaranteed. Defined terms are highlighted in the lexicons and corresponding definitions are entirely reported in the knowledge base as special contexts. After a manual clean-up phase due to the noise introduced by automatic tools, the average size of the four lexicons is about 1500 terms. The automatic construction of lexicons should be seen as a starting point for the setup of a dynamically growing resource. A manual update of the lexicons will be possible and should be done at a second stage directly by the users through terms insertion, deletion and inter-linguistic alignments editing.

\section{Building Domain Ontology}

The DALOS domain ontology is the result of an intellectual activity aimed at describing the domain of the consumer protection, chosen for the pilot case. It has been implemented as an extension of the Core Legal Ontology (CLO 4 [8] developed on top of DOLCE foundational ontology 9 and on the "Descriptions and Situations" (DnS) ontology [10] within the DOLCE+ library5. Such an extension is addressed to cope with the entities of the chosen domain and their legal specificities. In this knowledge architecture the role of a core legal ontology is to provide well established entities/concepts which belong to the general theory of law, bridging the gap between domain-specific concepts and the abstract categories of formal upper level or foundational ontologies such as,

\footnotetext{
4 http://www.loa-cnr.it/ontologies/CLO/CoreLegal.owl

5 DOLCE+ library, http://dolce.semanticweb.org
} 


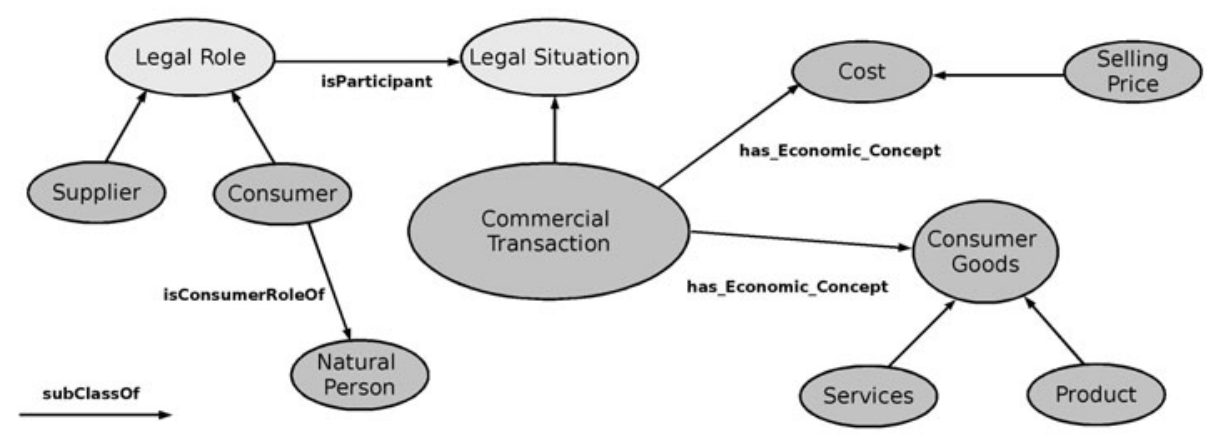

Fig. 1. Extract of the DALOS domain ontology

in our case, DOLCE. The domain ontology is therefore populated by the conceptual entities which characterize the consumer protection domain. The chosen approach in the ontology design has been to encode knowledge grounded on source text in order to avoid any forced concept harmonization. The first assumption is that all concepts that appear as definitions in the Dalos corpus are representative of the domain and, as a consequence, that several concepts used in the definitional contexts pertain to the ontology as well, representing the basic properties or, in other words, the 'intensional meaning' of the relevant concepts. Furthermore the domain Ontology contains generic situations having a legal relevance in the chosen domain. Such domain-specific concepts are classified according to more general notions, imported from CLO, as Legal_role and Legal_situation. An example of some concepts obtained by the definitions of the consumer law (as Commercial_ transaction, Consumer, Supplier, etc.) playing specific roles (10]) is given in Fig. 1. The first version of the Ontological layer contains 118 named classes.

\section{$5 \quad$ Integrating Resources}

One of the aim of the DALOS project was the construction of a knowledge base including ontologies and lexicons in different languages to be accessed in a flexible way in order to accomplish different tasks like drafting, consultation, document retrieving, assisted translation. In order to allow the integration of all the available resources in a single knowledge base, they have been chosen to be represented in the $\mathrm{RDF} / \mathrm{OWL}$ language.

\subsection{Lexicons}

For lexicon structure formalization, a meta-level ontology describing the WordNet semantic has been used. This is based on three classes: Synset, WordSense and Word. A WordNet synset is a set of one or more uninflected word forms (lemmas) with a synonymous meaning: for example trial, proceedings, law suit 


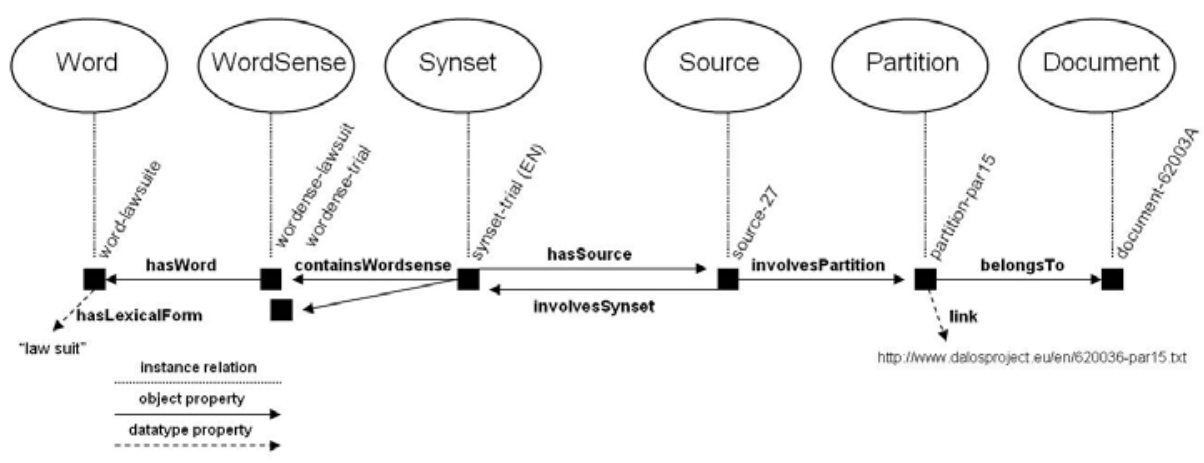

Fig. 2. Low level description of the English lexical resource "synset-trial" and one of its references to a EU directive

form a noun synset because they can be used to refer to the same concept. In this meta-level ontology the classes Synset, WordSense and Word and the properties relating instances of such classes are defined. Each Synset object is a set of WordSense objects since polysemous terms are distinct in wordsenses. In practice, each entry of a DALOS lexicon is represented as a synset object, i.e. a set of word objects in a particular meaning and one or more lexical forms associated (variants). This conversion from a WordNet like data structure in RDF is described at the W3C site 6 .

\subsection{Dalos Corpus}

Another meta-level ontology has been used to represent links, namely sources, between terms and document fragments in the DALOS corpus. Here the main classes are Document, Partition and Source whose instances are directly linked with instances of Synset. The Partition and Document classes represent the DALOS corpus fragments and documents and both have a datatype property specifying the location where the text can be accessed on-line. These two metalevel ontologies give a low level description of lexical resources and document fragments (Fig. 2).

\subsection{Concept Layer}

In order to integrate multilingual lexicons and references to documents with the domain ontology on Consumer Protection, a middle layer containing objects of type Concept has been implemented. These objects represent language independent concepts, i.e. objects representing a unique particular meaning that can be differently expressed in different languages. In a multilingual environment they can be seen as pivot entities that link the corresponding synsets through the hasLexicalization property. For this reason Concept objects identifiers (URI)

${ }^{6} \mathrm{RDF} / \mathrm{OWL}$ Representation of WordNet, http://www .w3.org/TR/wordnet-rdf/ 


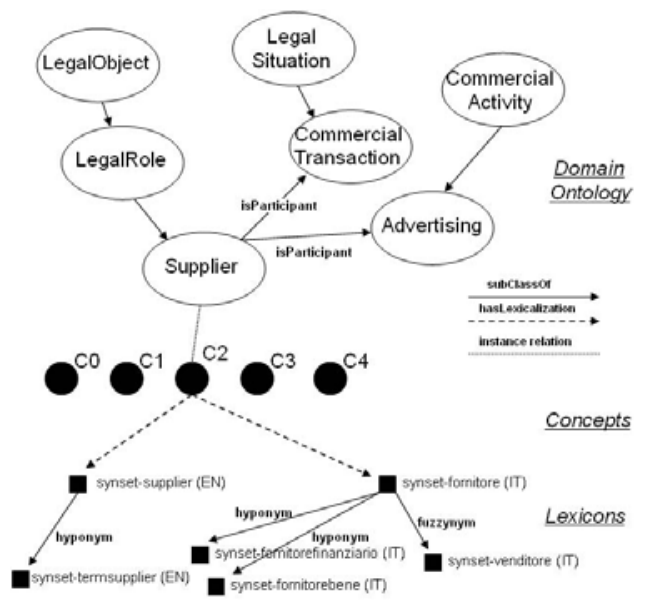

Fig. 3. Extract of the DALOS KB. The "C2" concept is classified in the ontology as a "Supplier" and has two lexicalizations for two different languages.

are language independent as shown in Fig. 3. The integration of lexicons with the domain ontology is then obtained through a classification of the concepts in the ontological classes. Moreover, on the Concept class, properties of partial matching like narrowMatch and broaderMatch are defined in order to implement relations of non perfect equivalence.

\subsection{Domain Ontology}

The Concept objects act as a sort of middle layer that mediates between linguistic aspects of terms and semantic/conceptual ones. It is then clear how synset resources in this knowledge base show both low level features deriving from their belonging to a lexicon in a specific language and invariant with respect to the domain, and high level feature deriving from their association to a concept object. In fact, when applying the domain ontology, concepts assume new roles and particular properties that are valid only in the Consumer Protection semantic sphere. Thus, for each synset it is possible to list both its linguistic relations, typically WordNet relations, and its semantic relations with other synsets in the same or in a different lexicon. Fig. 3 depicts most of the DALOS knowledge system, from ontology classes to lexical synsets. In this section it has been described how the DALOS knowledge base, a resource including ontologies, multilingual lexicons and documental corpus, has been designed and integrated. See also [11] for a theoretical foundation of heterogeneous knowledge resources integration. Particular attention has been paid to modularity, limiting as much as possible the coupling between lexicons and ontologies through the introduction of Concept objects middle layer in order to favour: 
- a modular use of the single knowledge components;

- maintenance and evolution;

- knowledge reuse and sharing;

- processability of the ontology by applications;

- collaborative ontology development;

- distributed reuse on the web.

In this view it is then possible for an application or an agent to exploit only the lexicons without applying the ontology and using only lexical properties and interlingual alignments. Otherwise it could be possible to select a single lexicon in order to work in a mono-lingual environment with or without sources. Many kind of applications can benefit of the availability of the described resources, for example an information retrieval system or a legal drafting application. This second case is described in the following section as an implemented prototype of use.

\section{The Application Prototype}

An application prototype has been developed within the project in order to show how the DALOS resource can be accessed and exploited to provide multilingual lexical and semantic support in legislative documents drafting concerning the "consumer protection" domain.

$x m$ LegesEdito $\sqrt{7}$ is an open source legislative drafting environment developed at ITTIG-CNR 12 for supporting the adoption of legal national standards (XML and URN NIR 8 standards). Briefly, xmLegesEditor is a visual XML editor able to support legislative drafters in the production of standard compliant normative documents, providing advanced features for structural and semantic markup. The DALOS extension of xmLegesEditor provides integrated access from the drafting environment to the knowledge resource produced in DALOS. Conforming to the modular structure of the knowledge model described in previous section, the application modules for accessing the resources have been designed to be able to selectively exploit the single components of the knowledge base in a dynamic and transparent way. The chosen language in the application for example, determines the lexicon to be loaded. Moreover, dealing with a large (and presumably increasing) amount of data, arises a number of interesting efficiency problems especially in a User Interactive application like an editing environment where response time has a crucial importance.

These have been faced by setting up solutions using:

- precalculation of needed inferences in order to limit to the least necessary runtime reasoning;

- an independent segmentation system for selective loading of data;

- a caching system for dynamically loading and disposing data.

This gave significant result in the application prototype integration in terms of time response and resources use.

\footnotetext{
7 http://www.xmleges.org

8 NormeInRete, http://www.normeinrete.it
} 


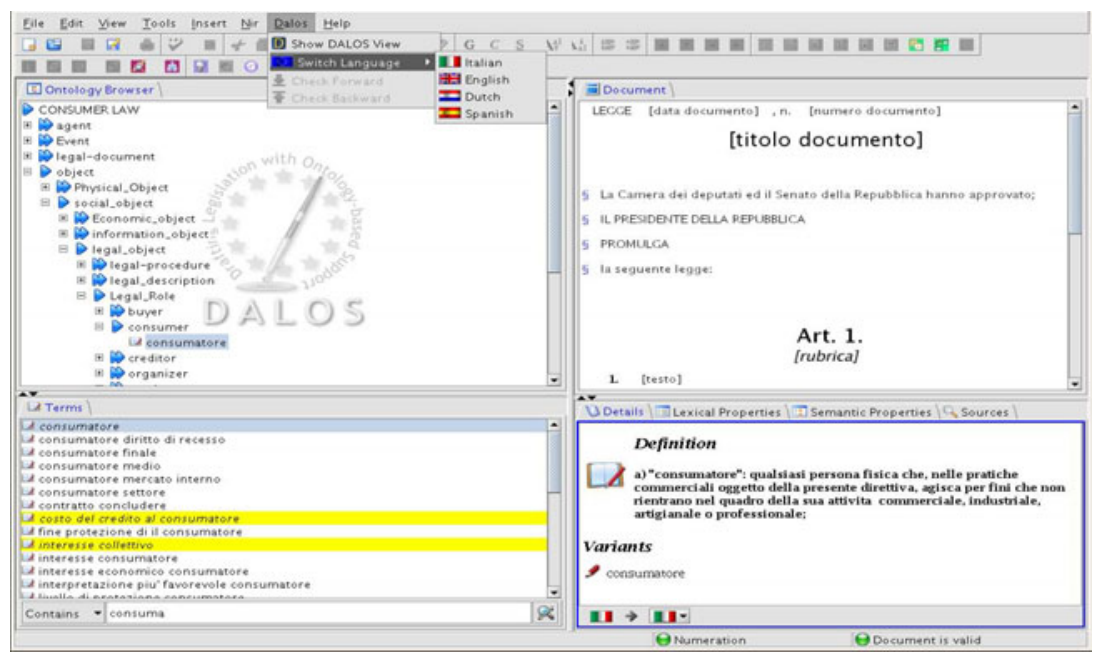

Fig. 4. A screenshot of xmLegesEditor DALOS prototype

\subsection{Accessing DALOS Resources within the Application Prototype}

Following its multiple components architecture a set of views on the Knowledge Base have been been implemented in the editing environment to provide access to the different resources (Fig. (4). Once the KB in a specific language is loaded in the application, users can access the controlled vocabulary from different perspectives exploiting

- terms classification accessed from a browsable hierarchical view of the ontology;

- direct search of terms in the lexicon from a plain list view from which the user can perform textual queries over the lexical forms of the extracted synsets

- access to detailed information over a synset as well as to hyperlinked external document fragments in the domain corpus where each term, as well as its variants, have been defined or used;

- view and browsing on sets of terms clustered according to their lexical relations (i.e. "hyponymy" , "fuzzynymy") or semantic relations i.e. relations inherited from the relations between the ontological classes under which each synset is classified (see sect. 5)

- interlingual relations provided by terms alignment for "horizontal" browsing in the multilingual resource

Starting form an initial core of loaded objects, additional information are dynamically loaded as needed following user interaction.

\section{Conclusions}

The main purpose of the DALOS project is to provide law-makers with linguistic and knowledge management tools to be used in the legislative processes, in 
particular within the phase of legislative drafting. The aim is to keep control over the legal language, especially in a multilingual environment, as the EU legislation one, enhancing the quality of the legislative production, as well as the accessibility and alignment of legislation at European level through explicit tagging of metainformation expressed in standard machine readable format.

In this paper the DALOS knowledge base design and implementation is presented, including NLP techniques used to create lexicons and their integration with a domain ontology. Finally the use of the DALOS resource in xmLegesEditor legislative drafting environment has been shown, along with facilities aiming at enhancing the quality of legislative texts.

The availability of a document archive marked up with a vocabulary of normalized terms derived by the DALOS resource can also be useful in documents indexing to provide enhanced retrieval services. Moreover, as terms in XML texts will be linked to the ontology, it will be possible to provide more advanced query features exploiting semantics for extracting norms or document fragments using more complex retrieval inferences.

The application prototype (the integrated environment composed by the drafting tool and the knowledge resource) has been tested and evaluated within the project by legislative offices of the Italian Parliament and CNIPA (Italian Minister of Reforms and Innovations in Public Administration / National Center for Information Technology in Public Administration) and by other public administration users in the Netherlands and Spain.

\section{References}

1. Mandelkern group on better regulation. final report, Tech. rep., European Commission (November 13, 2001)

2. Bizer, C., Heath, T., Berners-Lee, T.: Linked Data: The story so far. International Journal on Semantic Web \& Information Systems 5(3), 1-22 (2009)

3. Biasiotti, M., Francesconi, E., Palmirani, M., Sartor, G., Vitali, F.: Legal informatics and management of legislative documents. Tech. Rep. Working Paper No 2, Global Center for ICT in Parliament (2008)

4. Peristeras, V., Mentzas, G., Tarabanis, K.A., Abecker, A.: Transforming E-government and E-participation through IT 24(5), 14-19 (2009)

5. Dell'Orletta, F., Lenci, A., Marchi, S., Montemagni, S., Pirrelli, V.: Text2-knowledge: una piattaforma linguistico-computazionale per l'estrazione di conoscenza da testi. In: Proceedings of the SLI-2006 Conference, Vercelli, pp. 20-28 (2006)

6. Sclano, F., Velardi, P.: Termextractor: a web application to learn the shared terminology of emergent web communities. In: Proceedings of the 3rd International Conference on Interoperability for Enterprise Software and Applications, I-ESA 2007 (2007)

7. Cunningham, H., Maynard, D., Bontcheva, K., Tablan, V.: Gate: A framework and graphical development environment for robust nlp tools and applications. In: Proceedings of the 40th Anniversary Meeting of the Association for Computational Linguistics, ACL'02 (2002) 
8. Gangemi, A., Sagri, M., Tiscornia, D.: A constructive framework for legal ontologies. In: Benjamins, R., Casanovas, P., Gangemi, A., Selic, B. (eds.) Law and the Semantic Web. Springer, Heidelberg (2005)

9. Gangemi, A., Guarino, N., Masolo, C., Oltramari, A., Schneider, L.: Sweetening ontologies with dolce. In: Gómez-Pérez, A., Benjamins, V.R. (eds.) EKAW 2002. LNCS (LNAI), vol. 2473, p. 166. Springer, Heidelberg (2002)

10. Masolo, C., Vieu, L., Bottazzi, E., Catenacci, C., Ferrario, R., Gangemi, A., Guarino, N.: Social roles and their descriptions. In: Welty, C. (ed.) Proceedings of the Ninth International Conference on the Principles of Knowledge Representation and Reasoning, Whistler (2004)

11. Picca, D., Gliozzo, A., Gangemi, A.: LMM: an OWL-DL metamodel to represent heterogeneous lexical knowledge. In: Proceedings of LREC, Marrakech, Morocco, May 28-30 (2008)

12. Agnoloni, T., Francesconi, E., Spinosa, P.: xmLegesEditor: an opensource visual XML editor for supporting legal national standards. In: Proceedings of the V Legislative XML Workshop, pp. 239-251. European Press Academic Publishing (2007) 